\title{
Integration of transcriptomics, proteomics, metabolomics and systems pharmacology data to reveal the therapeutic mechanism underlying Chinese herbal Bufei Yishen formula for the treatment of chronic obstructive pulmonary disease
}

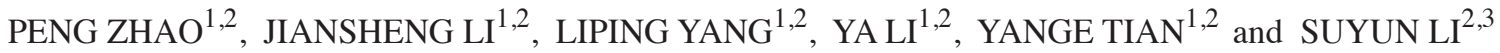 \\ ${ }^{1}$ Henan Key Laboratory of Chinese Medicine for Respiratory Disease; ${ }^{2}$ Collaborative Innovation Center for \\ Respiratory Disease Diagnosis and Treatment and Chinese Medicine Development of Henan Province, \\ Henan University of Chinese Medicine, Zhengzhou, Henan 450046; ${ }^{3}$ Department of Respiratory Diseases, \\ The First Affiliated Hospital of Henan University of Chinese Medicine, Zhengzhou, Henan 450000, P.R. China
}

Received July 14, 2016; Accepted January 4, 2018

DOI: $10.3892 / \mathrm{mmr} .2018 .8480$

\begin{abstract}
Bufei Yishen formula (BYF) is a traditional Chinese medicine formula, which has long been used as a therapeutic agent for the treatment of chronic obstructive pulmonary disease (COPD). Systems pharmacology has previously been used to identify the potential targets of BYF, and an experimental study has demonstrated that BYF is able to prevent COPD. In addition, the transcriptomic and metabolomic profiles of lung tissues from rats with COPD and BYF-treated rats have been characterized. The present study aimed to determine the therapeutic mechanisms underlying the effects of BYF on COPD treatment by integrating transcriptomics, proteomics and metabolomics, together with systems pharmacology datasets. Initially, the proteomic profiles of rats with COPD and BYF-treated rats were analyzed. Subsequently, pathway and network analyses were conducted to integrate three-omics data; the results demonstrated that the genes, proteins and metabolites were predominantly associated with oxidoreductase activity, antioxidant activity, focal adhesion and lipid metabolism. Finally, a comprehensive analysis of systems pharmacology, transcriptomic, proteomic and metabolomic datasets was performed, and numerous genes, proteins and metabolites were found to be regulated in BYF-treated rats; the potential target proteins of BYF were involved in lipid metabolism, inflammatory response, oxidative stress and focal adhesion. In conclusion, BYF exerted beneficial effects
\end{abstract}

Correspondence to: Dr Jiansheng Li, Henan Key Laboratory of Chinese Medicine for Respiratory Disease, Henan University of Chinese Medicine, 156 Jinshui Dong Road, Zhengzhou, Henan 450046, P.R. China

E-mail: 1i_js8@163.com

Key words: chronic obstructive pulmonary disease, Bufei Yishen formula, system pharmacology, transcriptome, proteome, metabolome against COPD, potentially by modulating lipid metabolism, the inflammatory response, oxidative stress and cell junction pathways at the system level.

\section{Introduction}

Chronic obstructive pulmonary disease (COPD) is a chronic inflammatory disease, which is characterized by progressive, partially reversible airflow limitation. COPD is considered the third most common life-threatening disease worldwide, and is associated with high morbidity and mortality $(1,2)$. In addition, COPD is considered to be not only a respiratory disease, but also a systemic disorder. Traditional Chinese medicine (TCM) formulas are comprehensive medicinal compounds that may provide a systemic approach to COPD therapy (3).

Bufei Yishen formula (BYF) is a TCM formula, which is composed of 12 medicinal herbs, that has long been used as a therapeutic agent for the treatment of COPD. In our previous clinical study, BYF was reported to exert beneficial effects on measured outcomes in patients with stable COPD over a 6-month treatment period and a 12-month follow-up period (4). Subsequently, a systems pharmacological model was constructed by integrating active compounds prediction, targets prediction and network pharmacology to identify 216 bioactive ingredients from BYF and 195 potential targets. Our previous study demonstrated that BYF was effective for the treatment of rats with COPD and ventricular hypertrophy, due to its inhibitory effects on the expression of inflammatory cytokines and hypertrophic factors, protease-antiprotease imbalance and collagen deposition in vivo (5). However, the systemic mechanism of BYF in the treatment of rats with COPD remains unclear. Therefore, the present study aimed to conduct a systems-level analysis of the therapeutic mechanism of BYF.

High-throughput molecularbiological techniques, including transcriptomic, proteomic and metabolomic approaches, have been used to explore complex biological processes and the function of TCM formulas in systems biology. Transcriptomic 
profiling is a promising approach to analyze the entire genome, which provides details regarding the biological processes underlying respiratory disease development and medical intervention (6). Proteomic profiling has been used to uncover the complexity of the therapeutic effects of TCM formulas by analyzing expressed proteins and protein function in a cellular context (7). Furthermore, metabolomic profiling provides data-rich information regarding the metabolic alterations that occur as a consequence of the transcriptome and proteome, which reflects the genetic, epigenetic, and environmental factors that influence cellular physiology (8). Therefore, combining transcriptomics, proteomics and metabolomics has the potential to provide a system-wide understanding of the complex therapeutic processes of TCM formulas $(9,10)$.

In our previous studies, the transcriptomic and metabolomic profiles of rats with COPD and BYF-treated rats were generated $(11,12)$. The present study aimed to further analyze the molecular mechanisms of BYF on rats with COPD using proteomic datasets. Subsequently, systems pharmacology, transcriptomics, proteomics and metabolomics datasets were integrated, with the aim of providing a system-wide understanding of the molecular mechanisms underlying the therapeutic effects of BYF on rats with COPD.

\section{Materials and methods}

Chemicals and animals. Klebsiella pneumoniae (strain ID: 46114) was obtained from the National Center for Medical Culture Collections (Beijing, China). Tobacco (Hongqi Canal ${ }^{\circledR}$ Filter tip cigarette; tobacco type: Tar, $10 \mathrm{mg}$; nicotine content, $1.0 \mathrm{mg}$; carbon monoxide, $12 \mathrm{mg}$ ) was purchased from China Tobacco Henan Industrial Co., Ltd. (Zhengzhou, China). A total of 32 Sprague-Dawley rats (16 male and 16 female; weight, 200 $\pm 20 \mathrm{~g}$; age, 6-8 weeks) were obtained from the Experimental Animal Center of Henan Province (Zhengzhou, China). The rats were housed in an animal room at a constant temperature $\left(25 \pm 2^{\circ} \mathrm{C}\right)$ under a 12 -h light/dark cycle with free access to food and water. The present study was approved by the Experimental Animal Care and Ethics Committee of The First Affiliated Hospital, Henan University of Traditional Chinese Medicine (Henan, China), and the methods were conducted in accordance with the approved guidelines of the Experimental Animal Care and Ethics Committee of The First Affiliated Hospital, Henan University of Traditional Chinese Medicine (register no. 2012HLD-0001).

COPD model and drug administration. The COPD rat model and BYF formula were prepared as previously described (13). Briefly, 22 rats (COPD group) were maintained in a closed box and were exposed to tobacco and repeated $K$. pneumoniae infections. The control group rats were untreated. At the end of week 8, two COPD rats were sacrificed for lung tissue collection, in order to validate that the rat model was successful. The herbal drugs contained within BYF were provided by the Department of Pharmacology, The First Affiliated Hospital, Henan University of Chinese Medicine, and were prepared in fluid extract. The components of BYF were as follows: Ginseng Radix et Rhizoma, 9 g; Astragali Radix, 15 g; Corni Fructus, 12 g; Lycii Fructus, 12 g; Schisandrae Chinensis Fructus, 9 g; Epimedii Herba, 9 g; Fritillariae Thunbergii Bulbus, 9 g;
Paeoniae Rubra Radix, 9 g; Pheretima, 12 g; Perillae Fructus, 9 g; Ardisiae Japonicae Herba, 15 g; and Citri Reticulatae Pericarpium, $9 \mathrm{~g}$ (5). On week 9, COPD rats were divided to two groups (10 rats each group) and intragastrically treated with normal saline $(2 \mathrm{ml})$ or BYF $(4.44 \mathrm{~g} / \mathrm{kg}, 0.5 \mathrm{~g} / \mathrm{ml})$ every day between weeks 9 and 20. The control group (10 rats) were also intragastrically treated with normal saline $(2 \mathrm{ml})$ for the same time period. On week 20, all rats were sacrificed, and lung tissues were collected.

Protein expression analysis. Proteins were isolated from the lung tissue from each of the three experimental groups. Briefly, the lung tissues were lysed in lysis buffer [4\% SDS, 0.1 M DTT, $0.1 \mathrm{M}$ Tris ( $\mathrm{pH}$ 8.0)] and homogenized using a mechanical homogenizer (Retsch Technology GmbH, Haan, Germany). The lysates were cleared by centrifugation at 12,000 x g and $4^{\circ} \mathrm{C}$ for $5 \mathrm{~min}$, prior to storage at $-80^{\circ} \mathrm{C}$ until further use. For proteolytic digestion, trypsin (Roche Diagnostics $\mathrm{GmbH}$, Mannheim, Germany) solution was added to the proteins and incubated for $24 \mathrm{~h}$ at $37^{\circ} \mathrm{C}$. Subsequently, each of the samples (30 $\mu \mathrm{l}$ ) was individually reconstituted with $70 \mu \mathrm{l}$ isopropanol, vortexed for $1 \mathrm{~min}$ at room temperature. Tryptic peptides were labeled with 8-plex isobaric tags (AB Sciex Germany $\mathrm{GmbH}$, Darmstadt, Germany) for relative quantitation according to the manufacturer's protocol.

Strong cation exchange fractionation was performed on a Shimadzu Prominence liquid chromatography system (Kyoto, Japan). Buffers A [10 mM KH $\mathrm{mH}_{4}$ in $25 \%$ acetonitrile (ACN); $\mathrm{pH} 3$ ] and $\mathrm{B}\left(10 \mathrm{mM} \mathrm{KH}_{2} \mathrm{PO}_{4}\right.$ and $2 \mathrm{M} \mathrm{KCl}$ in $25 \% \mathrm{ACN}$; $\mathrm{pH} 3$ ) were used as the mobile phase. The peptide mixtures were diluted 10 times with buffer $\mathrm{A}$ and $100 \mu \mathrm{l}$ was then loaded onto a PolySULFOETHYL A column $(5 \mu \mathrm{m} ; 100 \AA$; 100x4.6 mm i.d.; PolyLC, Columbia, MD, USA). The following gradients was used: $0-1 \mathrm{~min}, 0-5 \% \mathrm{~B} ; 1-21 \mathrm{~min}, 5-30 \% \mathrm{~B}$; 21-26 min, 30-50\% B; 26-31 min, 50\% B; 31-36 min, 50-100\% $\mathrm{B}$; $36-46 \mathrm{~min}, 100 \% \mathrm{~B}$. The flow rate was $1 \mathrm{ml} / \mathrm{min}$ and the column temperature was set at $35^{\circ} \mathrm{C}$. A total of 23 fractions (2 min/fraction) were collected and desalted with a C18 SPE column (Phenomenex, Torrance, CA, USA). The dried fractions were then dissolved in $0.1 \%$ formic acid (FA) for liquid chromatography (LC)-mass spectrometry (MS) analysis.

LC-tandem MS analysis was performed on a Prominence nano LC system (Shimadzu, Kyoto, Japan) coupled on-line to a micrOTOF-Q II mass spectrometer (Bruker Daltonik, Bremen, Germany). Water containing $0.1 \%$ FA, and ACN containing $0.1 \%$ FA were used as the mobile phases. The peptide samples ( $\sim 1 \mu \mathrm{g}$ of each fraction) were loaded onto a pulled tip column (15 cmx100 $\mu \mathrm{m}$ i.d.) packed with C18 Reprosil particles $(5 \mu \mathrm{m}$; Nikkyo Technos Co., Ltd., Tokyo, Japan). At the flow rate of $300 \mathrm{nl} / \mathrm{min}$, the gradient was as follows: 5-34\% B, $25 \mathrm{~min}$; 34-60\% B, 5 min; 60-80\% B, 5 min; $80 \%$ B, 4 min. Mass spectrometry analysis was operated in the positive mode and the ion source settings were as follows: spray voltage, $4500 \mathrm{~V}$; nebulizer pressure, $5 \mathrm{psi}$; desolvation gas temperature, $200^{\circ} \mathrm{C}$. and all MS and MS/MS spectra were obtained in data-dependent mode with one MS full-scan ranging from 300-1,800 m/z followed by $20 \mathrm{MS} / \mathrm{MS}$ scans.

The reporter ion ratio for each identified peptide was analyzed by Mascot (v2.2; Matrix Science, Inc., Boston, MA, USA). The proteomics data were analyzed by loess 

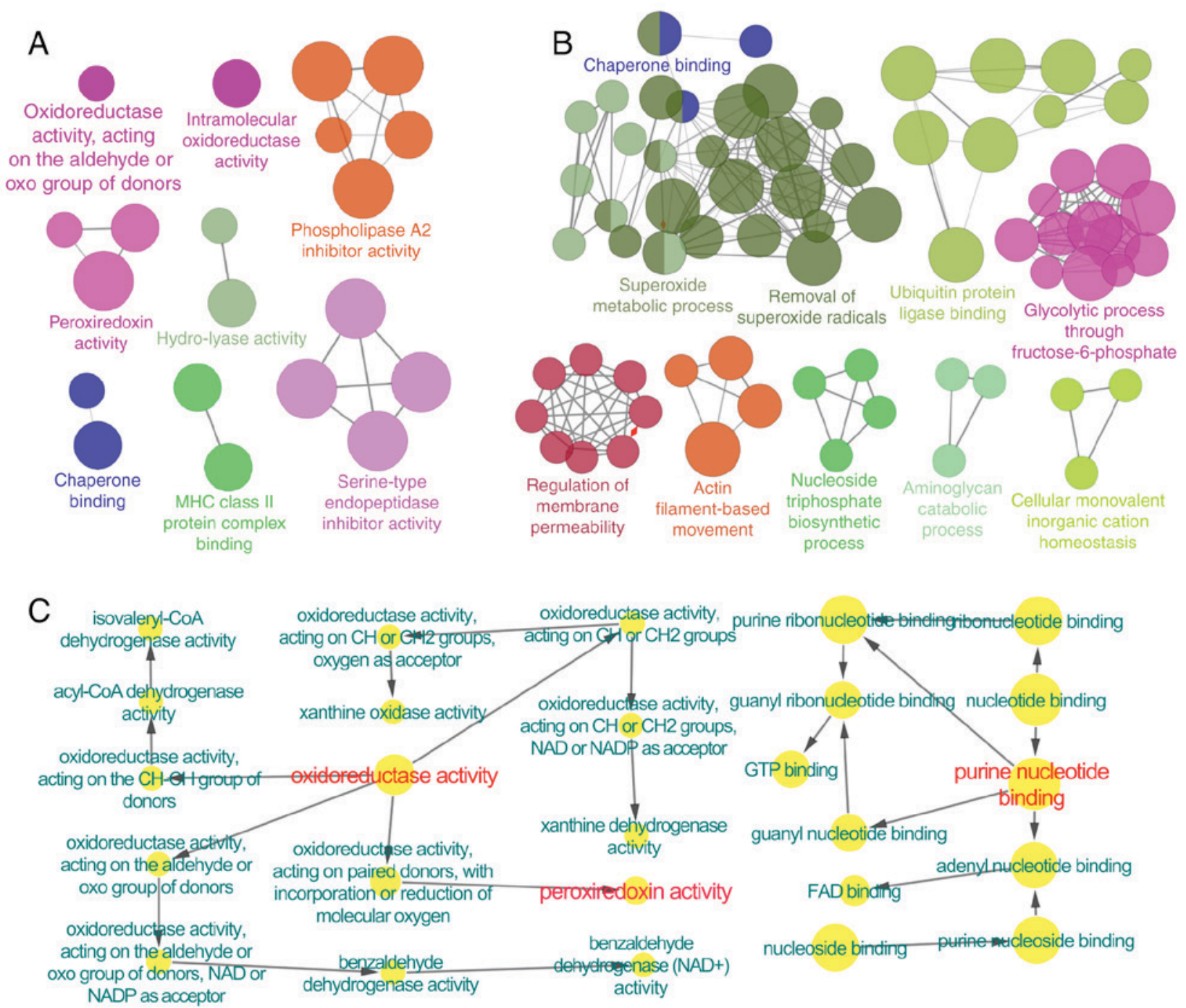

Figure 1. Molecular functions of regulated proteins in lung tissues from rats with COPD and BYF-treated rats. ClueGO was applied to analyze the molecular functions of the regulated proteins in (A) COPD rats and (B) BYF-treated rats. Functionally grouped networks of enriched categories were generated for the regulated proteins. In the functionally grouped networks, terms are presented as linked nodes, and functionally related groups partially overlap; node size represents the significance of term enrichment. (C) Molecular function of the overlapping proteins between COPD rats and BYF-treated rats was analyzed using BiNGO software. Node size is proportional to the number of proteins in the test set. Red indicates the predominant functions of the proteins and the molecular functions associated with BYF-therapeutic effect. BYF, Bufei Yishen formula; COPD, chronic obstructive pulmonary disease.

and global median normalization, and then underwent $\log 2$ transformation. All statistical analyses were performed using Student's t-test with the SPSS 19.0 software package (IBM Corp., Armonk, NY, USA). P $<0.05$ was considered to indicate a statistically significant difference. A fold-change $>1.0$ was considered upregulation, whereas a fold-change $<1.0$ was considered downregulation.

Gene/protein set enrichment, network and pathway analyses. The molecular function of proteins was explored using the Cytoscape v3.1.1 plugins ClueGO and BiNGO $(14,15)$. Pathway enrichment analysis of transcripts and proteins were analyzed using the Database for Annotation, Visualization and Integrated Discovery (https://david.ncifcrf.gov/) and Kyoto Encyclopedia of Genes and Genomes (KEGG; http://www.genome.jp/kegg/) database. Regulated pathways were considered statistically significant if the P-value was $\leq 0.05$. For correlation analyses on pathway levels, KEGG pathways, including the pathways of transcripts, proteins, target proteins of system pharmacology and metabolomics, were compared. Pathway enrichment analysis was performed using KEGG and the aforementioned pathways. In addition, Metscape was used to analyze the integrated pathway of gene, protein, target proteins of system pharmacology and metabolomics data (16).

\section{Results and Discussion}

Proteomic analysis results of BYF-treated COPD rats. In our previous study (5), systems pharmacology was used to identify the bioactive ingredients, and the potential targets of BYF. In addition, BYF treatment was confirmed to exert beneficial effects on rats with COPD, due to its inhibitory effects on inflammatory cytokine expression, protease-antiprotease imbalance and collagen deposition (5). Furthermore, we identified molecular alterations at the transcriptomic and metabolomic level $(11,12)$. To investigate the system-wide mechanism of BYF in COPD treatment, the present study 
Table I. Pathways associated with the proteins regulated in lung tissue from rats with chronic obstructive pulmonary disease.

\begin{tabular}{lccr}
\hline Term & Count & $\%$ & P-value \\
\hline Glycolysis/Gluconeogenesis & 10 & 0.4179 & $6.64 \times 10^{-6}$ \\
Hypertrophic cardiomyopathy & 9 & 0.3761 & $5.29 \times 10^{-5}$ \\
Dilated cardiomyopathy & 9 & 0.3761 & $8.70 \times 10^{-5}$ \\
Pyruvate metabolism & 6 & 0.2507 & $3.62 \times 10^{-4}$ \\
Glyoxylate and dicarboxylate metabolism & 4 & 0.1672 & $7.99 \times 10^{-4}$ \\
Tight junction & 9 & 0.3761 & 0.001079 \\
Citrate cycle (tricarboxylic acid cycle) & 5 & 0.2089 & 0.00125 \\
Leukocyte transendothelial migration & 8 & 0.3343 & 0.002374 \\
Focal adhesion & 10 & 0.4179 & 0.003903 \\
Tryptophan metabolism & 5 & 0.2089 & 0.004803 \\
Valine, leucine and isoleucine degradation & 5 & 0.2089 & 0.006122 \\
Adherens junction & 6 & 0.2507 & 0.00651 \\
Cardiac muscle contraction & 6 & 0.2507 & 0.008115
\end{tabular}

Table II. Pathways associated with the proteins regulated in lung tissue from Bufei Yishen formula-treated rats.

\begin{tabular}{lccr}
\hline Term & Count & $\%$ & P-value \\
\hline Focal adhesion & 13 & 0.5527 & $8.96 \times 10^{-5}$ \\
Tight junction & 10 & 0.4252 & $3.02 \times 10^{-4}$ \\
Hypertrophic cardiomyopathy & 8 & 0.3401 & $4.79 \times 10^{-4}$ \\
Regulation of actin cytoskeleton & 12 & 0.5102 & $6.92 \times 10^{-4}$ \\
Glycolysis/Gluconeogenesis & 7 & 0.2976 & 0.002728022 \\
Leukocyte transendothelial migration & 8 & 0.3401 & 0.003040578 \\
Dilated cardiomyopathy & 7 & 0.2976 & 0.003855419 \\
Adherens junction & 6 & 0.2551 & 0.007806313 \\
Extracellular matrix-receptor interaction & 6 & 0.2551 & 0.011328586 \\
Glyoxylate and dicarboxylate metabolism & 3 & 0.1276 & 0.016548236 \\
Metabolism of xenobiotics by cytochrome P450 & 5 & 0.2126 & 0.017848404 \\
Prion diseases & 4 & 0.1701 & 0.020435392 \\
Pyruvate metabolism & 4 & 0.1701 & 0.027198439 \\
Drug metabolism & 5 & 0.2126 & 0.032368833 \\
Fatty acid metabolism & 4 & 0.1701 & 0.032961744 \\
Tryptophan metabolism & 4 & 0.1701 & 0.035013439 \\
Valine, leucine and isoleucine degradation & 4 & 0.1701 & 0.041555659 \\
Cardiac muscle contraction & 5 & 0.2126 & 0.041625416 \\
\hline
\end{tabular}

examined the effects of BYF on the proteomic profiles of lung tissues.

Using an LC-MS-based proteomic analysis, 191 and 195 proteins were revealed to be regulated in the COPD model (vs. the control) and the BYF-treated rats (vs. the COPD model), respectively. According to a further analysis, the 191 proteins regulated in rats with COPD were predominantly associated with phospholipase A2 inhibitor activity, peroxiredoxin activity, oxidoreductase activity and major histocompatibility complex class II protein complex binding, etc. (Fig. 1A). In BYF-treated rats, the 198 regulated proteins were attributed to various molecular functions, including superoxide metabolic process, removal of superoxide radicals and glycolytic process through fructose-6-phosphate (Fig. 1B). These regulated proteins were involved in numerous pathways, including focal adhesion, tight junction, leukocyte transendothelial migration and regulation of actin cytoskeleton (Tables I and II).

Furthermore, the COPD model group (191 proteins) shared 98 common proteins with the BYF treated-group (195 proteins). Of these 98 proteins, the alterations in the expression of 61 proteins in the COPD model were suppressed by BYF treatment (Table III). These proteins were attributed to numerous biological functions and two pathways, including oxidoreductase activity, peroxiredoxin activity, purine nucleotide binding (Fig. 1C), as well as focal adhesion and the leukocyte transendothelial migration pathway (data not shown). 
Table III. Overlapping proteins between the chronic obstructive pulmonary disease and Bufei Yishen-treated groups.

\begin{tabular}{|c|c|c|c|c|c|}
\hline Accession number & Molecular weight (kDa) & $\log 2(\mathrm{~A} / \mathrm{B})$ & $\mathrm{A} / \mathrm{B}$ & $\log 2(\mathrm{~B} / \mathrm{F})$ & $\mathrm{B} / \mathrm{F}$ \\
\hline IPI00190577 & 404 & 0.1 & 1.071773 & -0.1 & 0.933033 \\
\hline IPI00191728 & 48 & 0.3 & 1.231144 & -0.1 & 0.933033 \\
\hline IPI00192301 & 22 & 0.1 & 1.071773 & -0.1 & 0.933033 \\
\hline IPI00193716 & 46 & 0.3 & 1.231144 & -0.2 & 0.870551 \\
\hline IPI00194097 & 54 & 0.3 & 1.231144 & -0.4 & 0.757858 \\
\hline IPI00195516 & 51 & 0.3 & 1.231144 & -0.2 & 0.870551 \\
\hline IPI00196994 & 23 & 0.3 & 1.231144 & -0.1 & 0.933033 \\
\hline IPI00197770 & 56 & -0.1 & 0.933033 & 0.1 & 1.071773 \\
\hline IPI00198887 & 57 & -0.3 & 0.812252 & 0.2 & 1.148698 \\
\hline IPI00200593 & 47 & 0.1 & 1.071773 & -0.2 & 0.870551 \\
\hline IPI00201300 & 44 & 0.1 & 1.071773 & -0.1 & 0.933033 \\
\hline IPI00201561 & 22 & 0.1 & 1.071773 & -0.1 & 0.933033 \\
\hline IPI00203214 & 95 & 0.2 & 1.148698 & -0.5 & 0.707107 \\
\hline IPI00205135 & 77 & 0.3 & 1.231144 & -0.2 & 0.870551 \\
\hline IPI00205332 & 35 & 0.8 & 1.741101 & -0.5 & 0.707107 \\
\hline IPI00206403 & 38 & -0.3 & 0.812252 & 0.4 & 1.319508 \\
\hline IPI00207014 & 45 & 0.1 & 1.071773 & -0.2 & 0.870551 \\
\hline IPI00207146 & 16 & -0.2 & 0.870551 & 0.3 & 1.231144 \\
\hline IPI00208422 & 88 & 0.3 & 1.231144 & -0.3 & 0.812252 \\
\hline IPI00209113 & 226 & -0.1 & 0.933033 & 0.1 & 1.071773 \\
\hline IPI00211448 & 61 & -0.1 & 0.933033 & 0.2 & 1.148698 \\
\hline IPI00212314 & 68 & 0.4 & 1.319508 & -0.3 & 0.812252 \\
\hline IPI00212523 & 20 & -0.6 & 0.659754 & 0.2 & 1.148698 \\
\hline IPI00214457 & 21 & -0.3 & 0.812252 & 0.3 & 1.231144 \\
\hline IPI00215564 & 24 & 0.1 & 1.071773 & -0.1 & 0.933033 \\
\hline IPI00230787 & 29 & -0.3 & 0.812252 & 0.5 & 1.414214 \\
\hline IPI00231423 & 64 & -0.1 & 0.933033 & 0.5 & 1.414214 \\
\hline IPI00231643 & 16 & 0.1 & 1.071773 & -0.3 & 0.812252 \\
\hline IPI00231694 & 146 & 0.3 & 1.231144 & -0.5 & 0.707107 \\
\hline IPI00231825 & 16 & 0.2 & 1.148698 & -0.2 & 0.870551 \\
\hline IPI00231925 & 41 & -0.5 & 0.707107 & 1.1 & 2.143547 \\
\hline IPI00324986 & 51 & 0.1 & 1.071773 & -0.4 & 0.757858 \\
\hline IPI00325189 & 17 & 0.3 & 1.231144 & -0.1 & 0.933033 \\
\hline IPI00326140 & 167 & -0.5 & 0.707107 & 0.3 & 1.231144 \\
\hline IPI00326179 & 50 & 0.3 & 1.231144 & -0.1 & 0.933033 \\
\hline IPI00326972 & 62 & 0.5 & 1.414214 & -0.2 & 0.870551 \\
\hline IPI00327502 & 26 & -0.2 & 0.870551 & 0.2 & 1.148698 \\
\hline IPI00337168 & 58 & 0.6 & 1.515717 & -0.3 & 0.812252 \\
\hline IPI00358087 & 37 & -0.2 & 0.870551 & 0.3 & 1.231144 \\
\hline IPI00360930 & 28 & -0.4 & 0.757858 & 0.8 & 1.741101 \\
\hline IPI00362072 & 45 & -0.2 & 0.870551 & 0.2 & 1.148698 \\
\hline IPI00362755 & 204 & -0.1 & 0.933033 & 0.4 & 1.319508 \\
\hline IPI00363395 & 21 & 0.2 & 1.148698 & -0.1 & 0.933033 \\
\hline IPI00364890 & 42 & 0.6 & 1.515717 & -0.4 & 0.757858 \\
\hline IPI00365929 & 49 & -0.1 & 0.933033 & 0.6 & 1.515717 \\
\hline IPI00368347 & 118 & 0.8 & 1.741101 & -0.4 & 0.757858 \\
\hline IPI00370654 & 29 & 0.3 & 1.231144 & -0.3 & 0.812252 \\
\hline IPI00371990 & 27 & -0.3 & 0.812252 & 0.2 & 1.148698 \\
\hline IPI00372839 & 110 & 0.3 & 1.231144 & -0.1 & 0.933033 \\
\hline IPI00373505 & 39 & -0.2 & 0.870551 & 0.3 & 1.231144 \\
\hline IPI00388249 & 80 & -0.6 & 0.659754 & 0.6 & 1.515717 \\
\hline IPI00389571 & 54 & 0.1 & 1.071773 & -0.1 & 0.933033 \\
\hline
\end{tabular}


Table III. Continued.

\begin{tabular}{lcccrc}
\hline Accession number & Molecular weight $(\mathrm{kDa})$ & $\log 2(\mathrm{~A} / \mathrm{B})$ & $\mathrm{A} / \mathrm{B}$ & $\log 2(\mathrm{~B} / \mathrm{F})$ & $\mathrm{B} / \mathrm{F}$ \\
\hline IPI00392216 & 68 & 0.1 & 1.071773 & -0.1 & 0.933033 \\
IPI00411230 & 26 & -0.1 & 0.933033 & 0.2 & 1.148698 \\
IPI00421428 & 29 & 0.4 & 1.319508 & -0.4 & 0.757858 \\
IPI00421517 & 53 & -0.1 & 0.933033 & 0.2 & 1.148698 \\
IPI00470288 & 43 & 0.3 & 1.231144 & -0.1 & 0.933033 \\
IPI00471584 & 83 & -0.1 & 0.933033 & 0.1 & 1.071773 \\
IPI00480679 & -0.7 & 0.615572 & 0.4 & 1.319508 \\
IPI00948384 & 14 & 0.4 & 1.319508 & -0.2 & 0.870551 \\
IPI01016479 & 375 & 0.7 & 1.624505 & -0.1 & 0.933033 \\
\hline
\end{tabular}

A

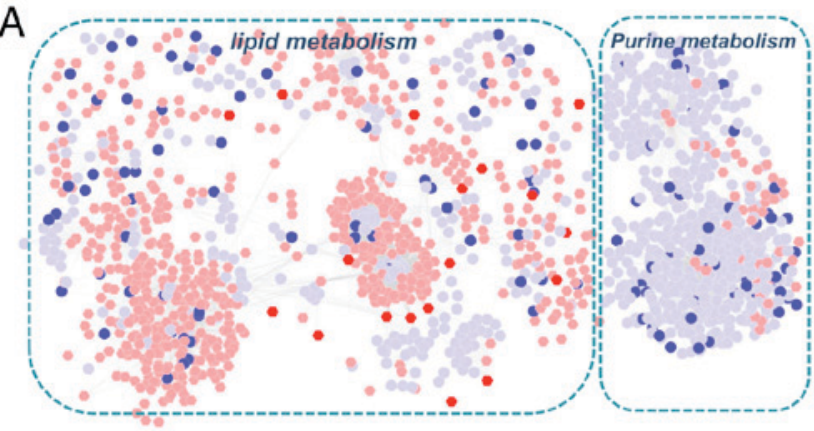

$\mathrm{B}$

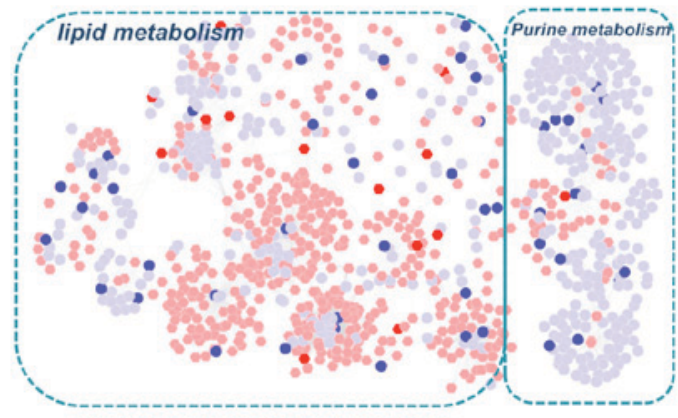

C

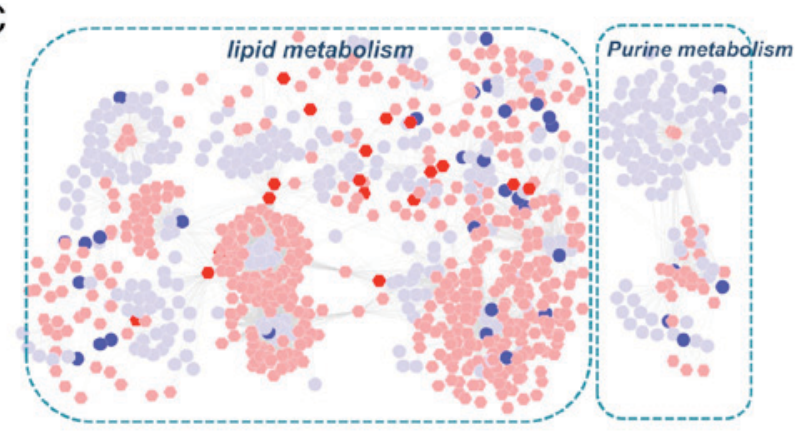

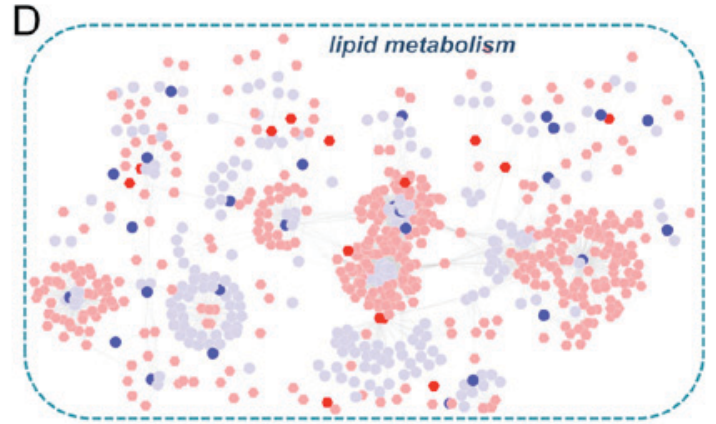

Figure 2. Correlation analysis of the metabolites, genes and proteins regulated in lung tissues of COPD rats and BYF-treated rats. Compound reaction networks of the metabolites, genes and proteins were visualized using Metscape: Metabolites (hexagons) and metabolic enzymes (circles) are presented as nodes and reactions are presented as edges. Inputted genes and proteins are shown in red, inputted metabolites are shown in blue. (A) Metabolite-gene network of the COPD model group. (B) Metabolite-gene network of the BYF-treated group. (C) Metabolite-protein network of the COPD model group. (D) Metabolite-protein network of the BYF-treated group. BYF, Bufei Yishen formula; COPD, chronic obstructive pulmonary disease.

Association between genes, proteins and metabolites. In our previous study, 18 samples were randomly chosen from three experimental groups for gene expression experiments. According to a cut-off value of $\mathrm{P}<0.05,2,463$ and 2,292 differentially expressed genes were detected between the control and COPD groups, and the COPD and BYF-treated groups, respectively (11). In addition, 49 and 31 regulated metabolites were detected in lung tissues from COPD rats and BYF treated-rats, respectively (12). The present study aimed to provide a system-wide view of the therapeutic mechanism of BYF in COPD treatment by integrating transcriptomics, proteomics and metabolomics data.

Metscape software was used to investigate the latent relationships between the gene, protein and metabolite measurements. Initially, two gene-metabolite networks were generated based on the transcriptomics and metabolomics data of COPD and BYF-treated rats. As shown in Fig. 2A and B, the metabolite-gene networks were mainly associated with lipid and purine metabolism. Subsequently, protein-metabolite networks were generated using the metabolomics and proteomics data from COPD and BYF-treated rats (Fig. 2C and $\mathrm{D})$. The results demonstrated that these proteins and metabolites were also predominantly associated with lipid and purine metabolism. Furthermore, the majority of metabolites were involved in lipid metabolism in the gene/protein-metabolite networks. These findings suggested that lipid metabolism may be the critical biological process associated with COPD development and medical intervention.

Comprehensive analysis of systems pharmacology, transcriptomics, proteomics and metabolomics data. Systems 
A

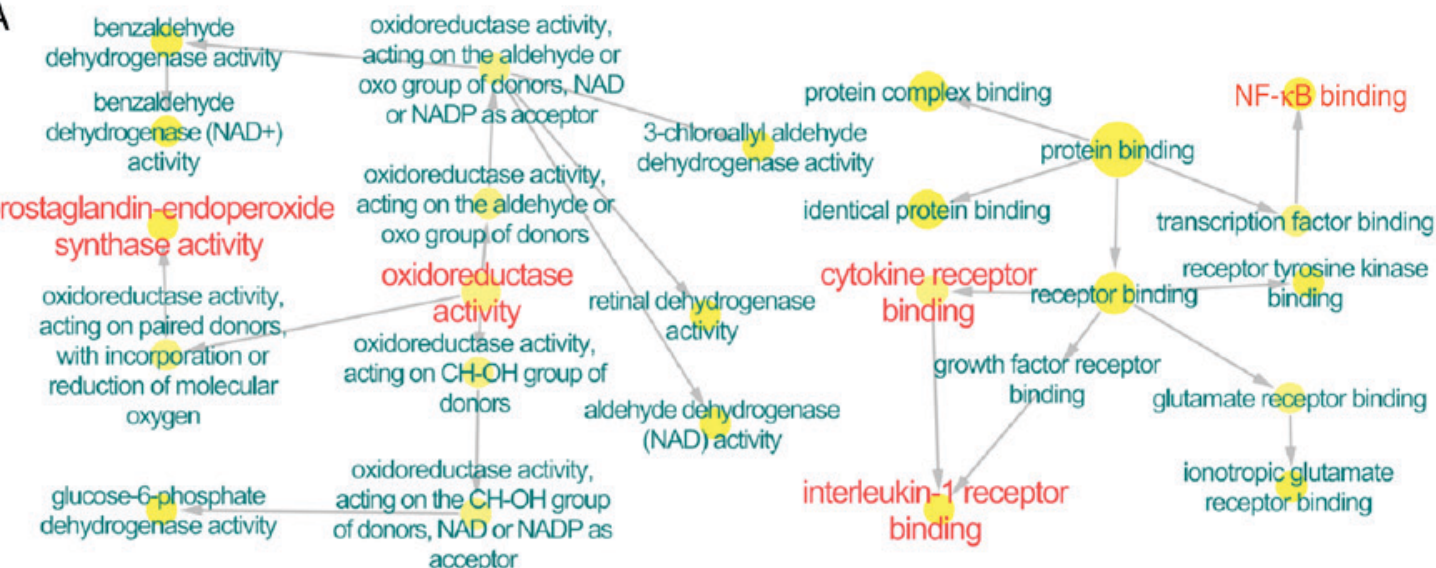

B

\begin{tabular}{|c|c|c|}
\hline xanthine oxidase activity & $\begin{array}{l}\text { oxidoreductase activity, acting } \\
\text { on } \mathrm{CH} \text { or } \mathrm{CH} 2 \text { groups, oxygen } \\
\text { as acceptor }\end{array}$ & $\begin{array}{l}\text { xanthine dehydrogenase } \\
\text { activity }\end{array}$ \\
\hline $\begin{array}{l}\text { aldehyde dehydrogenase } \\
\text { (NAD) activity }\end{array}$ & $\begin{array}{l}\text { oxidoreductase activity, acting } \\
\text { on } \mathrm{CH} \text { or } \mathrm{CH} 2 \text { groups }\end{array}$ & $\begin{array}{c}\text { oxidoreductase activity, acting } \\
\text { on } \mathrm{CH} \text { or } \mathrm{CH} 2 \text { groups, NAD or } \\
\mathrm{NADP} \text { as acceptor }\end{array}$ \\
\hline $\begin{array}{l}\text { 3-chloroallyl aldehyde } \\
\text { dehydrogenase activity }\end{array}$ & oxidoreductase activity & $\begin{array}{c}\text { oxidoreductase activity, acting } \\
\text { on superoxide radicals as } \\
\text { acceptor }\end{array}$ \\
\hline tinal dehydrogenase activit. & $\begin{array}{l}\text { xidoreductase activity, acting } \\
\text { on the aldehyde or oxo group } \\
\text { of donors }\end{array}$ & superoxide dismutase activity \\
\hline $\begin{array}{l}(N A D+) \text { activity } \\
\text { senzaldehyde dehydrogenase } \\
\text { activity }\end{array}$ & $\begin{array}{c}\text { oxidoreductase activity, acting } \\
\text { on the aldehyde or oxo group } \\
\text { of donors, NAD or NADP as } \\
\text { acceptor }\end{array}$ & antioxidant activity \\
\hline
\end{tabular}

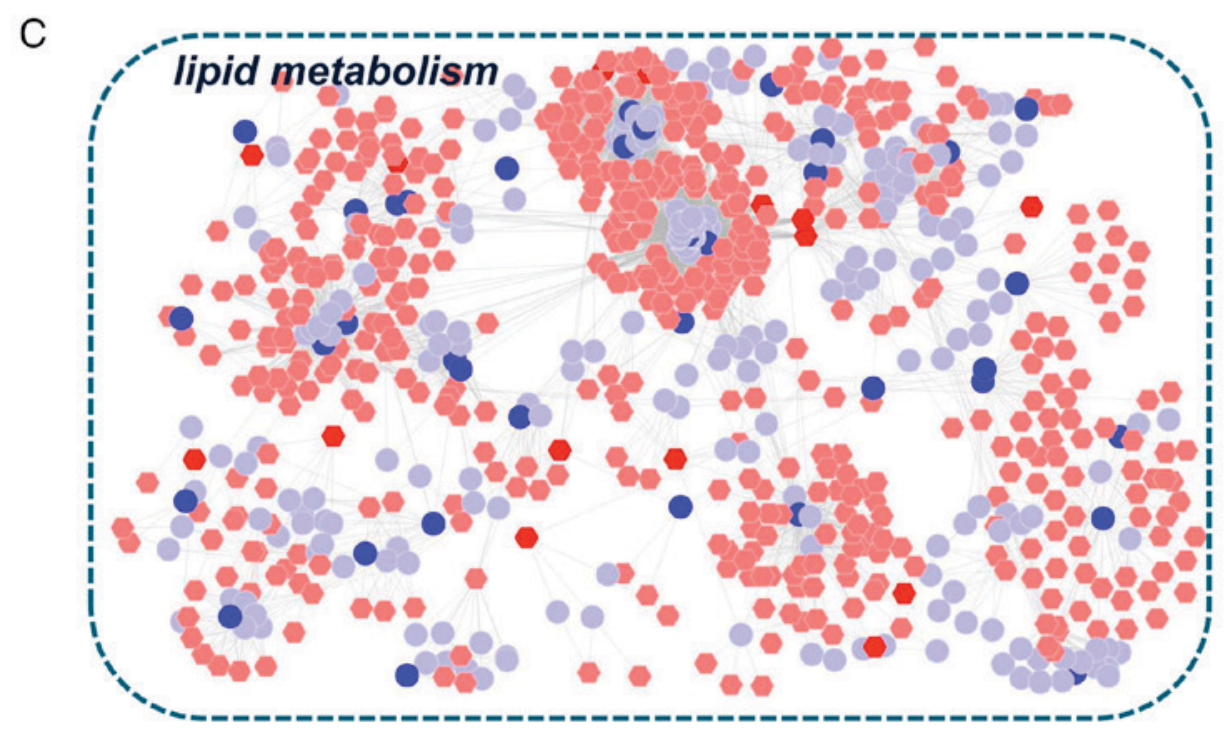

Figure 3. Molecular functions of the overlapping proteins, and the correlation between the metabolites regulated in BYF-treated rats and target proteins. The molecular functions of the overlapping proteins were analyzed using BiNGO. The area of a node was proportional to the number of proteins in the test set. (A) Molecular function of overlapping proteins between the potential targets and transcript measurements in lung tissues of BYF-treated rats. (B) Molecular function of overlapping proteins between the potential targets and proteome measurements in lung tissues of BYF-treated rats. Red indicates the predominant functions of the proteins, and the molecular functions associated with BYF-therapeutic effect. (C) Metscape software was used to build a compound reaction network: Metabolites (hexagons) and metabolic enzymes (circles) are presented as nodes and reactions are presented as edges. Inputted metabolites are shown in blue, inputted target proteins are shown in red. BYF, Bufei Yishen formula.

pharmacology was previously applied to identify the active compounds and potential targets of BYF (5). To provide a more in-depth understanding of the systemic mechanism of BYF in treating COPD rats, the systems pharmacology, transcriptomics, proteomics and metabolomics data were integrated.

Initially, the direct correlation between the potential targets and transcripts were analyzed. A total of 10 overlapping 
Lipid metabolism
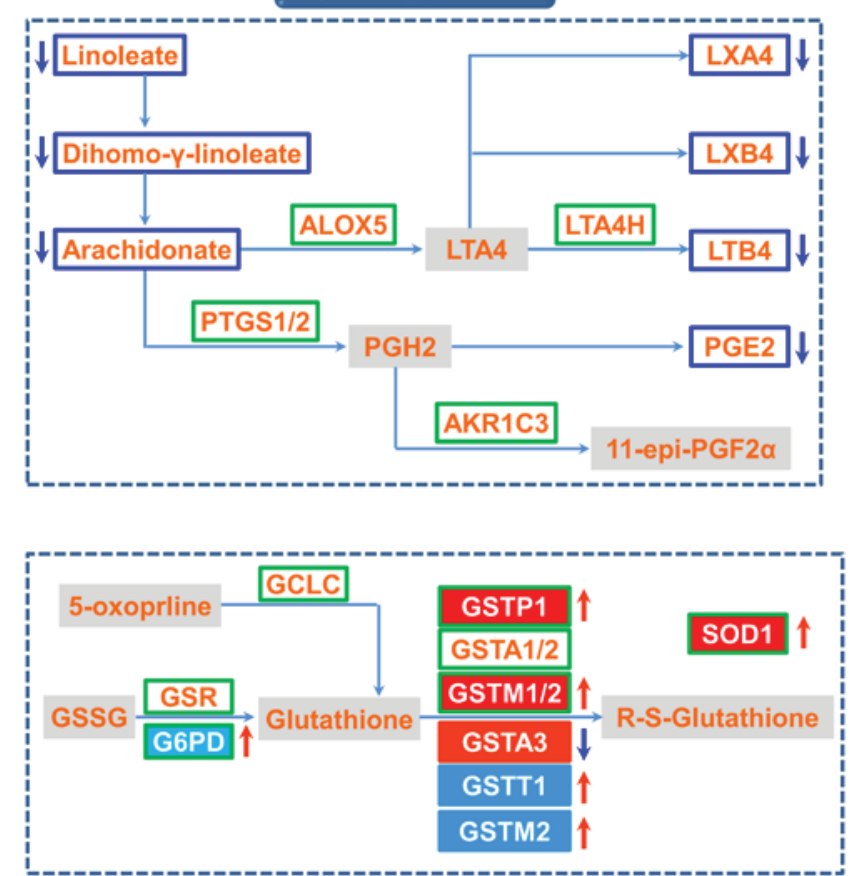

Oxidative stress
Inflammatory response
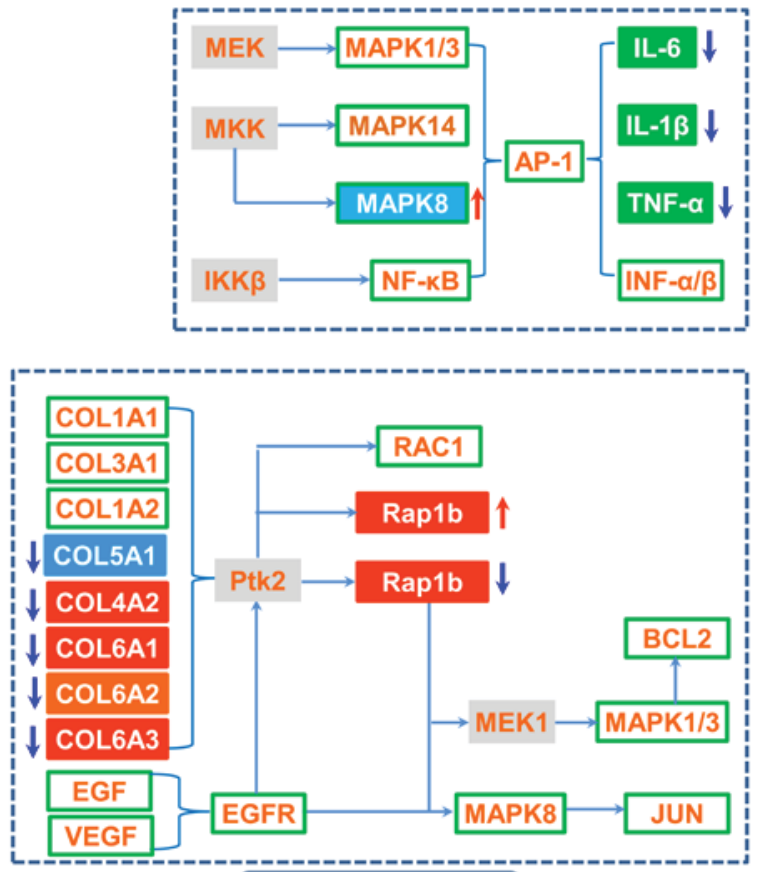

Focal adhesion

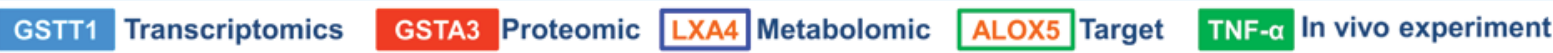

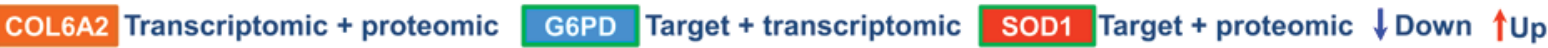

Figure 4. Comprehensive analysis of the potential targets of BYF, and the regulated genes, proteins and metabolites in the lung tissues of BYF-treated rats. The potential targets, genes, proteins and metabolites are presented as different-colored rectangles. Regulation is color-coded: Red arrow indicates upregulation, blue arrow indicates downregulation, and a gray rectangle represents no regulation. BYF, Bufei Yishen formula.

proteins [aldehyde dehydrogenase, mitochondrial (ALDH2); cell division protein kinase 4; checkpoint kinase 1; dopamine receptor D2; glucose-6-phosphate 1-dehydrogenase (G6PD); glutathione S-transferase Mu (GSTM)2; interleukin (IL)-1 $\beta$; vascular endothelial growth factor receptor 2; glucocorticoid receptor; prostaglandin $\mathrm{G} / \mathrm{H}$ synthase (PTGS)1] between the targets of BYF and the transcripts regulated in the BYF-treated group were detected, which could be attributed to various molecular functions, including oxidoreductase activity, nuclear factor $(\mathrm{NF})-\kappa \mathrm{B}$ binding and IL-1 receptor binding (Fig. 3A). Subsequently, 8 overlapping proteins [GSTM1; GSTM2; glutathione S-transferase P; superoxide dismutase (Cu-Zn) (SOD1); xanthine dehydrogenase/oxidase; 78 kDa glucose-regulated protein; ALDH2; ATP synthase subunit $\beta$, mitochondrial] were identified between the potential targets and the proteins regulated in BYF-treated rats (Fig. 3B). These proteins were primarily involved in oxidoreductase and antioxidant activity. Subsequently, the latent correlation between the target proteins and metabolites regulated in the BYF-treated group was determined using Metscape software (Fig. 3C). The results demonstrated that the metabolites and target proteins were predominantly involved in lipid metabolism.

Based on the integrated analysis, a comprehensive image of the therapeutic mechanism of BYF in COPD treatment was generated (Fig. 4). The comprehensive image predominantly consisted of four groups: Lipid metabolism, inflammatory response, oxidative stress and focal adhesion.

Our previous study demonstrated that BYF achieved its ameliorative effect on rats with COPD by suppressing the expression of inflammatory cytokines, including IL-1 $\beta$, IL-6 and tumor necrosis factor- $\alpha$ (5). The systems pharmacology results indicated that extracellular signal-regulated kinase (ERK), p38, c-Jun N-terminal kinase (JNK) and $\mathrm{NF}-\kappa \mathrm{B}$ were the potential targets of the active compounds contained in BYF. These data suggested that BYF may decrease the expression levels of inflammatory cytokines, potentially by regulating the activation of ERK, p38, JNK and $\mathrm{NF}-\kappa \mathrm{B}$.

In our metabolomics study, it was demonstrated that arachidonic acid metabolism was a significantly dysregulated pathway; with the metabolites linoleate, dihomo- $\gamma$-linoleate, lipoxin (LX)A4, LXB4, leukotriene (LT)B4, and prostaglandin (PG)E2, decreased by BYF treatment (12). Specifically, LXA4, LXB4, LTB4 and PGE2 participated in inflammatory processes in the airways of patients with COPD $(17,18)$, and metabolic enzymes, including arachidonate 5-lipoxygenase, PTGS1/2, LTA4 hydrolase and aldo-keto reductase family 1 member $\mathrm{C} 3$, were potential targets of BYF. Therefore, these findings 
Table IV. Associated pathways of the potential targets of Bufei Yishen formula.

\begin{tabular}{|c|c|c|c|}
\hline Term & Count & $\%$ & P-value \\
\hline Neuroactive ligand-receptor interaction & 30 & 0.7413 & $\mathrm{P}<0.0001$ \\
\hline Amyotrophic lateral sclerosis & 12 & 0.2965 & $\mathrm{P}<0.0001$ \\
\hline Pathways in cancer & 27 & 0.6672 & $\mathrm{P}<0.0001$ \\
\hline Drug metabolism & 12 & 0.2965 & $\mathrm{P}<0.0001$ \\
\hline Calcium signaling pathway & 19 & 0.4695 & $\mathrm{P}<0.0001$ \\
\hline Bladder cancer & 10 & 0.2471 & $\mathrm{P}<0.0001$ \\
\hline Metabolism of xenobiotics by cytochrome P450 & 11 & 0.2718 & $\mathrm{P}<0.0001$ \\
\hline Non-small cell lung cancer & 10 & 0.2471 & $\mathrm{P}<0.0001$ \\
\hline Glutathione metabolism & 9 & 0.2224 & 0.0001 \\
\hline Colorectal cancer & 11 & 0.2718 & 0.0001 \\
\hline Small cell lung cancer & 11 & 0.2718 & 0.0001 \\
\hline Pancreatic cancer & 10 & 0.2471 & 0.0001 \\
\hline Prostate cancer & 11 & 0.2718 & 0.0001 \\
\hline Vascular endothelial growth factor signaling pathway & 10 & 0.2471 & 0.0002 \\
\hline Gap junction & 10 & 0.2471 & 0.0007 \\
\hline Thyroid cancer & 6 & 0.1483 & 0.001 \\
\hline Focal adhesion & 15 & 0.3706 & 0.0011 \\
\hline Gonadotropin-releasing hormone signaling pathway & 10 & 0.2471 & 0.0013 \\
\hline Glioma & 8 & 0.1977 & 0.0015 \\
\hline Alzheimer's disease & 13 & 0.3212 & 0.0015 \\
\hline Progesterone-mediated oocyte maturation & 9 & 0.2224 & 0.0022 \\
\hline Prion diseases & 6 & 0.1483 & 0.0024 \\
\hline T cell receptor signaling pathway & 10 & 0.2471 & 0.0026 \\
\hline Arginine and proline metabolism & 7 & 0.173 & 0.003 \\
\hline Melanoma & 8 & 0.1977 & 0.003 \\
\hline Fc epsilon RI signaling pathway & 8 & 0.1977 & 0.0051 \\
\hline Toll-like receptor signaling pathway & 9 & 0.2224 & 0.0061 \\
\hline Neurotrophin signaling pathway & 10 & 0.2471 & 0.0065 \\
\hline Nucleotide-binding oligomerization domain-like receptor signaling pathway & 7 & 0.173 & 0.0065 \\
\hline Apoptosis & 8 & 0.1977 & 0.0093 \\
\hline Adipocytokine signaling pathway & 7 & 0.173 & 0.0095 \\
\hline Vascular smooth muscle contraction & 9 & 0.2224 & 0.0111 \\
\hline Insulin signaling pathway & 10 & 0.2471 & 0.0111 \\
\hline Renal cell carcinoma & 7 & 0.173 & 0.0117 \\
\hline Endometrial cancer & 6 & 0.1483 & 0.0132 \\
\hline Mitogen-activated protein kinase signaling pathway & 15 & 0.3706 & 0.0137 \\
\hline Caffeine metabolism & 3 & 0.0741 & 0.0142 \\
\hline B cell receptor signaling pathway & 7 & 0.173 & 0.0161 \\
\hline Arachidonic acid metabolism & 6 & 0.1483 & 0.0178 \\
\hline Graft-versus-host disease & 5 & 0.1235 & 0.021 \\
\hline Phenylalanine metabolism & 4 & 0.0988 & 0.021 \\
\hline Tryptophan metabolism & 5 & 0.1235 & 0.0228 \\
\hline Oocyte meiosis & 8 & 0.1977 & 0.0302 \\
\hline ErbB signaling pathway & 7 & 0.173 & 0.031 \\
\hline Tyrosine metabolism & 5 & 0.1235 & 0.0312 \\
\hline p53 signaling pathway & 6 & 0.1483 & 0.0374 \\
\hline Epithelial cell signaling in Helicobacter pylori infection & 6 & 0.1483 & 0.0374 \\
\hline Type II diabetes mellitus & 5 & 0.1235 & 0.0385 \\
\hline Complement and coagulation cascades & 6 & 0.1483 & 0.0395 \\
\hline
\end{tabular}


indicated that BYF achieved its anti-inflammatory activity probably through suppressing lipid metabolism, including arachidonic acid metabolism.

Oxidative stress can trigger sustained inflammatory responses and is the major contributing factor to obstructive lung disorders $(19,20)$. In the present study, 8 overlapping proteins were identified between the targets of BYF and proteomic measurements of the BYF-treated group, which were predominantly involved in oxidative stress. For example, the potential targets (glutamate-cysteine ligase catalytic subunit; glutathione reductase, mitochondrial; G6PD; glutathione S-transferase P; glutathione S-transferase A1/2; GSTM1/2), were involved in glutathione metabolism $(21,22)$. In addition, the levels of antioxidant proteins, including SOD1, which were involved in the pathogenesis of COPD, were increased by BYF treatment $(23,24)$. These results suggested that regulating oxidative stress status may be one of the main causes of the anti-inflammatory activity of BYF.

In addition, focal adhesion, which is an overlapping pathway among proteomic measurements and the potential targets of BYF (Tables II and IV), was significantly regulated by BYF treatment. Specifically, the activation of mitogen-activated protein kinase $1 / 3$ and 8 , and JUN, which are potential targets of BYF, results in upregulation of the transcript levels of preinflammatory cytokines (25-27). Taken together, the present study demonstrated that BYF provided therapeutic benefits against COPD through modulating numerous biological functions, including lipid metabolism, oxidative stress, inflammatory response and focal adhesion pathway.

Our previous systems pharmacology study identified the active compounds and potential targets of BYF, and demonstrated that BYF treatment was able to exert therapeutic effects against rats with COPD (5). In addition, the transcriptomic and metabolomic profiles of lung tissues derived from COPD and BYF-treated rats were generated. In the present study, a systems biology approach was used to analyze the therapeutic mechanism of BYF in treating COPD rats. Initially, proteomic profiles were obtained from the lung tissues of COPD and BYF-treated rats, and the three levels of omics data were then integrated. The gene/protein-metabolite model was generated from the regulated genes, proteins and metabolites, and was used to identify the affected pathways and to examine them according to the measured abundances of genes, proteins and metabolites. The results indicated that these transcripts, proteins and metabolites were attributed to various functions, including oxidoreductase activity, antioxidant activity and lipid metabolism. Subsequently, a comprehensive method was used to integrate the systems pharmacology and 3 -omics data. The system-wide findings demonstrated that BYF potentially achieved its therapeutic effects over COPD rats by regulating lipid metabolism, the inflammatory response, oxidative stress and focal adhesion pathways at the systems level. In conclusion, the present study suggested that an integrated systems pharmacology and transcriptomics, proteomics and metabolomics approach has the potential to considerably advance understanding regarding the therapeutic mechanism of TCM.

\section{Acknowledgements}

The present study was supported by the National Natural Science Fund of China (grant no. 81130062).

\section{Competing interests}

The authors declare that they have no competing interests.

\section{References}

1. Vestbo J,Hurd SS,Agustí AG,Jones PW,VogelmeierC,Anzueto A, Barnes PJ, Fabbri LM, Martinez FJ, Nishimura M, et al: Global strategy for the diagnosis, management, and prevention of chronic obstructive pulmonary disease GOLD executive summary. Am J Respir Crit Care Med 187: 347-365, 2013.

2. Beran D, Zar HJ, Perrin C, Menezes AM and Burney P; Forum of International Respiratory Societies working group collaboration: Burden of asthma and chronic obstructive pulmonary disease and access to essential medicines in low-income and middle-income countries. Lancet Resp Med 3: 159-170, 2015.

3. Gan WQ, Man SF, Senthilselvan A and Sin DD: Association between chronic obstructive pulmonary disease and systemic inflammation: A systematic review and a meta-analysis. Thorax 59: 574-580, 2004.

4. Li SY, Li JS, Wang MH, Xie Y, Yu XQ, Sun ZK, Ma LJ, Zhang W, Zhang HL, Cao F and Pan YC: Effects of comprehensive therapy based on traditional Chinese medicine patterns in stable chronic obstructive pulmonary disease: A four-center, open-label, randomized, controlled study. BMC Complement Altern Med 12: 197, 2012.

5. Li J, Zhao P, Li Y, Tian Y and Wang Y: Systems pharmacology-based dissection of mechanisms of Chinese medicinal formula Bufei Yishen as an effective treatment for chronic obstructive pulmonary disease. Sci Rep 5: 15290, 2015.

6. Su G, Burant CF, Beecher CW, Athey BD and Meng F: Integrated metabolome and transcriptome analysis of the NCI60 dataset. BMC Bioinformatics 12 (Suppl 1): S36, 2011.

7. Vogel C and Marcotte EM: Insights into the regulation of protein abundance from proteomic and transcriptomic analyses. Nat Rev Genet 13: 227-232, 2012.

8. Tan KC, Ipcho SV, Trengove RD, Oliver RP and Solomon PS: Assessing the impact of transcriptomics, proteomics and metabolomics on fungal phytopathology. Mol Plant Pathol 10: 703-715, 2009.

9. Wilmes A, Limonciel A, Aschauer L, Moenks K, Bielow C, Leonard MO, Hamon J, Carpi D, Ruzek S, Handler A, et al: Application of integrated transcriptomic, proteomic and metabolomic profiling for the delineation of mechanisms of drug induced cell stress. J Proteomics 79: 180-194, 2013.

10. Meierhofer D, Weidner C and Sauer S: Integrative analysis of transcriptomics, proteomics, and metabolomics data of white adipose and liver tissue of high-fat diet and rosiglitazone-treated insulin-resistant mice identified pathway alterations and molecular hubs. J Proteome Res 13: 5592-5602, 2014.

11. Li J, Yang L, Yao Q, Li Y, Tian Y, Li S, Jiang S, Wang Y, Li X and Guo Z: Effects and mechanism of bufei yishen formula in a rat chronic obstructive pulmonary disease model. Evid Based Complement Alternat Med 2014: 381976, 2014.

12. Yang LP, Li JS, Li Y, Tian Y, Li S, Jiang S, Wang Y and Song X: Identification of metabolites and metabolic pathways related to treatment with Bufei Yishen formula in a Rat COPD model using HPLC Q-TOF/MS. Evid-Based Complement Alternat Med 2015: $956750,2015$.

13. Li Y, Li SY, Li JS, Deng L, Tian YG, Jiang SL, Wang Y and Wang YY: A rat model for stable chronic obstructive pulmonary disease induced by cigarette smoke inhalation and repetitive bacterial infection. Biol Pharm Bull 35: 1752-1760, 2012.

14. Maere S, Heymans K and Kuiper M: BiNGO: A Cytoscape plugin to assess overrepresentation of gene ontology categories in biological networks. Bioinformatics 21: 3448-3449, 2005.

15. Bindea G, Mlecnik B, Hackl H, Charoentong P, Tosolini M, Kirilovsky A, Fridman WH, Pagès F, Trajanoski Z and Galon J: ClueGO: A Cytoscape plug-in to decipher functionally grouped gene ontology and pathway annotation networks. Bioinformatics 25: 1091-1093, 2009. 
16. Karnovsky A, Weymouth T, Hull T, Tarcea VG, Scardoni G, Laudanna C, Sartor MA, Stringer KA, Jagadish HV, Burant C, et al: Metscape 2 bioinformatics tool for the analysis and visualization of metabolomics and gene expression data. Bioinformatics 28: 373-380, 2012.

17. Santus P, Sola A, Carlucci P, Fumagalli F, Di Gennaro A, Mondoni M, Carnini C, Centanni S and Sala A: Lipid peroxidation and 5-lipoxygenase activity in chronic obstructive pulmonary disease. Am J Respir Crit Care Med 171: 838-843, 2005.

18. Tulah AS, Parker SG, Moffatt MF, Wardlaw AJ, Connolly MJ and Sayers I: The role of ALOX5AP, LTA4H and LTB4R polymorphisms in determining baseline lung function and COPD susceptibility in UK smokers. BMC Med Genet 12: 173, 2011.

19. Barnes PJ: Cellular and molecular mechanisms of chronic obstructive pulmonary disease. Clin Chest Med 35: 71-86, 2014.

20. Sunnetcioglu A, Alp HH, Sertogullarindan B, Balaharoglu R and Gunbatar H: Evaluation of oxidative damage and antioxidant mechanisms in COPD, lung cancer, and obstructive sleep apnea syndrome. Respir Care 61: 205-211, 2016.

21. Lakhdar R, Denden S, Mouhamed MH, Chalgoum A, Leban N, Knani J, Lefranc G, Miled A, Ben Chibani J and Khelil AH: Correlation of EPHX1, GSTP1, GSTM1, and GSTT1 genetic polymorphisms with antioxidative stress markers in chronic obstructive pulmonary disease. Exp Lung Res 37: 195-204, 2011.

22. Escribano A, Amor M, Pastor S, Castillo S, Sanz F, Codoñer-Franch P and Dasí F: Decreased glutathione and low catalase activity contribute to oxidative stress in children with $\alpha-1$ antitrypsin deficiency. Thorax 70: 82-83, 2015.
23. Rahman I and MacNee W: Antioxidant pharmacological therapies for COPD. Curr Opin Pharmacol 12: 256-265, 2012.

24. Harju T, Kaarteenaho-Wiik R, Sirvio R, Sirviö R, Pääkkö P, Crapo JD, Oury TD, Soini Y and Kinnula VL: Manganese superoxide dismutase is increased in the airways of smokers' lungs. Eur Respir J 24: 765-771, 2004.

25. Dong X, Liu Y, Du M, Wang Q, Yu CT and Fan X: P38 mitogen-activated protein kinase inhibition attenuates pulmonary inflammatory response in a rat cardiopulmonary bypass model. Eur J Cardiothorac Surg 30: 77-84, 2006

26. Huang Y, Meng XM, Jiang GL, Yang YR, Liu J, Lv XW and Li J: Studies on mitogen-activated protein kinase signaling pathway in the alveolar macrophages of chronic bronchitis rats. Mol Cell Biochem 400: 97-105, 2015.

27. Kida Y, Kobayashi M, Suzuki T, Takeshita A, Okamatsu Y, Hanazawa S, Yasui T and Hasegawa K: Interleukin-1 stimulates cytokines, prostaglandin E2 and matrix metalloproteinase-1 production via activation of MAPK/AP-1 and NF-kappaB in human gingival fibroblasts. Cytokine 29: 159-168, 2005.

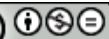

This work is licensed under a Creative Commons Attribution-NonCommercial-NoDerivatives 4.0 International (CC BY-NC-ND 4.0) License. 